\title{
Construction of random perfect phylogeny matrix
}

This article was published in the following Dove Press journal:

Advances and Applications in Bioinformatics and Chemistry

15 November 2010

Number of times this article has been viewed

\section{Mehdi Sadeghi ${ }^{1,2}$ \\ Hamid Pezeshk ${ }^{4}$ \\ Changiz Eslahchi ${ }^{3,5}$ \\ Sara Ahmadian ${ }^{6}$ \\ Sepideh Mah Abadi ${ }^{6}$}

'National Institute of Genetic Engineering and Biotechnology, Tehran, Iran; ${ }^{2}$ School of Computer Science, ${ }^{3}$ School of Mathematics, Institute for Research in Fundamental Sciences (IPM), Tehran, Iran; ${ }^{4}$ School of Mathematics, Statistics and Computer Sciences, Center of Excellence in Biomathematics, College of Science, University of Tehran, Tehran, Iran; ${ }^{5}$ Department of Mathematics, Shahid Beheshti University, G.C., Tehran, Iran; ${ }^{6}$ Department of Computer Engineering, Sharif University of Technology, Tehran, Iran
Correspondence: Changiz Eslahchi Department of Mathematics, Shahid Beheshti University, G.C., Tehran, Iran $\mathrm{Tel} / \mathrm{Fax}+982122431652$

Email ch-eslahchi@sbu.ac.ir
Purpose: Interest in developing methods appropriate for mapping increasing amounts of genome-wide molecular data are increasing rapidly. There is also an increasing need for methods that are able to efficiently simulate such data.

Patients and methods: In this article, we provide a graph-theory approach to find the necessary and sufficient conditions for the existence of a phylogeny matrix with $k$ nonidentical haplotypes, $n$ single nucleotide polymorphisms (SNPs), and a population size of $m$ for which the minimum allele frequency of each SNP is between two specific numbers $a$ and $b$.

Results: We introduce an $O\left(\max \left(n^{2}, n m\right)\right)$ algorithm for the random construction of such a phylogeny matrix. The running time of any algorithm for solving this problem would be $\Omega(\mathrm{nm})$.

Conclusion: We have developed software, RAPPER, based on this algorithm, which is available at http://bioinf.cs.ipm.ir/softwares/RAPPER.

Keywords: perfect phylogeny, minimum allele frequency (MAF), tree, recursive algorithm

\section{Introduction}

With the widespread availability of molecular data, computational methods for gene mapping are being developed. Often, the statistical properties and the behavior of these methods need to be assessed and tested by simulation. By increasing the number of computational methods for gene mapping, there is an increasing need for tools that can simulate data for long genomic regions. One of the most popular models used to infer haplotypes from genotype data is perfect phylogeny. ${ }^{1-3}$ This model reconstructs haplotype sequences with the assumptions of infinite sites and no recombination. Given a set of genotypes, the goal is to find a set of haplotypes that fit a perfect phylogeny. The solution divides haplotypes into disjoint blocks that are all compatible with a perfect phylogeny tree. Each block can be seen as a region of genome with different evolutionary history. Simulation of genotype or haplotype data based on a coalescent model is central to estimation methods and testing new methodologies. Coalescent simulation can be used to understand the statistical properties of DNA sequences under different evolutionary scenarios and also evaluate and compare different methods for haplotype analysis. A number of simulation programs have been developed under this model and are currently being used. ${ }^{4-11}$ We suggest a haplotype simulation to produce haplotype data with predefined allele frequencies with coalescent property. By using the set of haplotypes that satisfy the coalescent property, we can simulate a long genomic region, which can be used as an approximation to the evolutionary process that produce the real data. This simulation constructs a random perfect phylogeny matrix (PPM) with $k$ 
nonidentical haplotypes, $n$ single nucleotide polymorphisms (SNPs), and a population size of $m$ in which the minimum allele frequency (MAF) of each SNP is between two predefined numbers. A simulated data set for generating a long DNA sequence can be constructed based on assumptions about recombination rate and distribution in an evolutionary model among these perfect phylogeny blocks. The phylogenetic tree is represented by a matrix $A$ in which $a_{i j}$ is the state of character $j$ in sequence $i$, and the $i$ th row is the character vector of sequence $i$. In this article, we assume that characters are binary and directed, ie, only $0 \rightarrow 1$ changes may occur on any path from the root to a leaf of the tree. For the output, the ancestral state of an allele is represented by zero. We suggest a haplotype simulation approach that produces haplotype data with prespecified allele frequencies that satisfy coalescent model, ie, it produces a phylogenetic tree in which branches model the changes through the time of evolution based on the model. By above discussions, finding a method to construct random PPM with $k$ nonidentical haplotypes, $n$ SNPs, and a population size of $m$ in which the MAF of each SNP is between two specific numbers $a$ and $b$ will be very useful for data simulation (We consider MAF of column $c$ in $A$ as the number of 1 's in column $c$.) In this article, we take a graph-theory approach to the problem and show that there is a one-to-one correspondence between the set of perfect phylogeny matrices with certain conditions and some rooted trees. We find the necessary and sufficient conditions for the existence of such trees with respect to input parameters. We present an $O\left(\max \left(n^{2}, n m\right)\right)$ algorithm for generating a random matrix with the above conditions. We have developed a software based on this algorithm, RAPPER, which generates these matrices in a reasonable time. This article is organized as follows: we provide preliminaries and formulate the problem; in sections 3 and 4, Matrices and trees, and Extended tree following Gusfield, ${ }^{12}$ we construct a tree for every matrix and discuss its properties. In Necessary conditions, we discuss the necessary conditions. In Sufficient conditions, we find some sufficient conditions and present an algorithm to generate a random sample of the abovementioned matrices.

\section{Preliminaries}

To find some necessary and sufficient conditions for the existence of a PPM with $m$ rows, $k$ nonidentical rows, and $n$ columns such that in every column, number of 1 s are between $a$ and $b$, we need some definitions. We consider the cases that $k \geq 2$.

Definition 1. Let $a$ and $b$ be 2 integers and assume $a \leq b$. The matrix $B_{m \times n}$ is called a $(k, a, b)$-PPM if
1. $B$ is a PPM

2. The number of 1 's in each column is between $a$ and $b$

3. $B$ has $k$ nonidentical rows

Example 1. The following matrix $B$ is a $(3,2,3)-P P M$.

$$
B=\left[\begin{array}{lll}
1 & 1 & 0 \\
1 & 1 & 0 \\
0 & 0 & 1 \\
0 & 0 & 1 \\
1 & 0 & 0
\end{array}\right]
$$
if

Definition 2. The matrix $A_{k \times n}$ is called (m,a,b)-extendable

1. $A$ is a PPM

2. $A$ has $k$ nonidentical rows

3. There exists a matrix $B_{m \times n}$ that is a $(k, a, b)-\mathrm{PPM}$, and the rows of $A$ and $B$ are identical. (In this case, we say that $A_{k \times n}$ is extendable to $B_{m \times n}$.)

Example $2 A$ is $(5,2,3)$-extendable to $B$.

$$
A_{3 \times 3}=\left[\begin{array}{lll}
1 & 1 & 0 \\
1 & 0 & 0 \\
0 & 0 & 1
\end{array}\right] \rightarrow B_{5 \times 3}=\left[\begin{array}{ccc}
1 & 1 & 0 \\
1 & 1 & 0 \\
0 & 0 & 1 \\
0 & 0 & 1 \\
1 & 0 & 0
\end{array}\right]
$$

It is obvious that there exists a $(k, a, b)$-PPM matrix $B_{m \times n}$ if and only if there exists an $(m, a, b)$-extendable matrix $A_{k \times n}$.

Definition 3. A matrix $B$ is called good if it can be decomposed as follows:

1. The entries of its leftmost column are all 1's.

2. There exist good matrices $B_{1}, B_{2}, \ldots, B_{d}$ such that the rest ( 0 or more) of the columns of $B$ form the block structure, as illustrated in Figure 1.

Definition 4. A matrix $A$ is called canonical if it satisfies the second condition of the good matrix definition.

In the following definition, we assign a root to each good matrix.

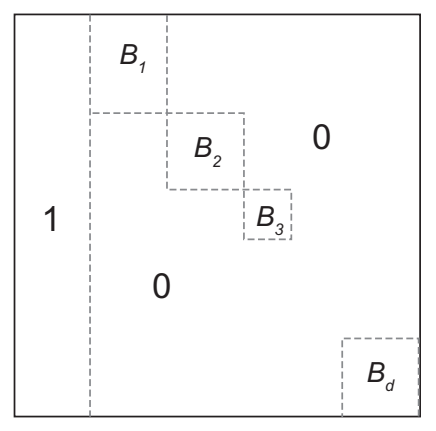

Figure I Block structure of a good matrix. 
Definition 5. In a good matrix $B$, we consider the leftmost all-one column as the root of the $B$.

\section{Matrices and trees Constructing a tree from a matrix}

According to Theorem 8 of Pe'er et al, ${ }^{13}$ every PPM has an ordering of its rows and columns, which yields a canonical matrix.

Theorem (Pe'er et al). ${ }^{13}$ Let $B$ be a binary matrix. The following are equivalent:

1. $B$ has a phylogenetic tree.

2. There exists an ordering of the rows and columns of $B$, which yields a canonical matrix.

Let $A_{k \times n}$ be a PPM that consists of $B_{l}, B_{2}, \ldots, B_{d}$ good blocks and $c_{i}$ be the corresponding root of $B_{i}$. Following Gusfield, ${ }^{12}$ we construct a labeled tree $T_{(A)}$ by using the following steps (see Figure 2):

$$
A=\left(\begin{array}{lll}
1 & 2 & 3 \\
1 & 1 & 0 \\
1 & 0 & 0 \\
0 & 0 & 1
\end{array}\right)
$$

Add an all-one-column to $\mathrm{A}$.

$$
B_{1}
$$

$$
A=\left(\begin{array}{ccccc}
0 & 1 & 2 & 3 \\
1 & 1 & 1 & 0 \\
1 & 1 & 0 & 0 \\
1 & 1 & 0 & 0 & 1
\end{array}\right) B_{2}
$$

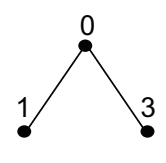

Construct a tree for $B_{2}$ of $A$ :

$$
\mathrm{B}_{2}=\left(\begin{array}{l}
3 \\
1
\end{array}\right)
$$

Construct a tree for $B_{1}$ of $A$ :
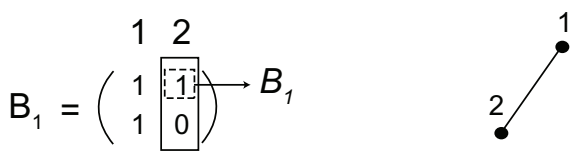

Construct a tree for block $B_{1}$ of above matrix :

$B_{1}=\left(\begin{array}{l}2 \\ 1\end{array}\right)$

Tree constructed from A :

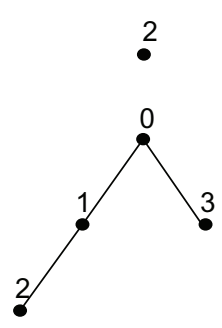

Figure 2 Constructing a labeled tree TA from a perfect phylogenetic matrix, A.
1. Add an all-one column to $A$ as the leftmost column. Index this column by 0 .

2. Let vertex 0 be the parent of $c_{i}$ for all $1 \leq i \leq d$.

3. Construct a tree from canonical form of every $B_{i}$ in a recursive manner. (Note that $B_{i}$ is a good matrix and has an all-one column.)

The vertex set of $T_{(A)}$ is $\{0,1,2, \ldots, n\}$. Now, we label some vertices of $T_{(A)}$ as follows:

If the last 1 entry in row $r$ occurs in column $j$, then we label vertex $j$ of $T_{(A)}$ with $r$. Gusfield ${ }^{12}$ proved the following lemma.

Lemma 1. Every leaf of $T_{(A)}$ is labeled, and every vertex is labeled at most once.

\section{Necessary and sufficient conditions for PPM}

Let $A$ be a matrix and $O_{v}=\left\{r / a_{r v}=1\right\}$. According to Estabrook et al ${ }^{14}$ and Meacham, ${ }^{15} A$ is PPM if and only if for every two columns $u$ and $v, O_{u} \cap O_{v}=\phi$ or $O_{u} \subset O_{v}$ or $O_{v} \subset O_{u}$.

Lemma 2. Let $r$ be a row in $A$ and $v$ be a vertex in $T_{(A)}$ with label $r$; then $a_{r u}=1$ if and only if $u$ is located in a path from root to $v$.

Proof. Let $u$ be located in a path from root to $v$. So $u$ is an ancestor of $v$. By the way that the tree was constructed from canonical form of matrix $A$, we have $O_{v} \subset O_{u}$. So, if $a_{r v}=1$ then $a_{r u}=1$.

Now, let $a_{r u}=1$. Since $v$ is labeled by $r$, the last nonzero entry of $r$ occurs in $v$. So, for every child of $v$ such as $w$, we have $a_{r w}=0$. Therefore, $a_{r u}=1$ implies that $u$ is not a child of $v$. As $a_{r u}=a_{r v}=1, O_{u} \cap O_{v} \neq \phi$. Thus, $O_{v} \subset O_{u}$ or $O_{u} \subset O_{v}$. Since $u$ is not a child of $v, O_{u}$ is not a subset of $O_{v}$. So, we have $O_{v} \subset O_{u}$, and $u$ is an ancestor of $v$. Therefore, $u$ is located in a path from root to $v$.

\section{Constructing a matrix from the tree}

Definition 6. A rooted tree $T$ is called a $(k, n)$-complete tree if it satisfies the following conditions:

1. $V(T)=\{0,1,2, \ldots, n\}$.

2. For every $1 \leq i \leq k$, there exists exactly 1 vertex with label $i$.

3. Every leaf is labeled.

4. Every vertex has at most 1 label.

From Lemma 1 and the way we construct $T_{(A)}$, we obtain that $T_{(A)}$ is $(k, n)$-complete tree where $A_{k \rtimes n}$ is a PPM. Now, for every $(k, n)$-complete tree $T$, we construct a PPM $A_{k x n}^{T}$ with nonidentical rows as follows:

Let $c$ be an arbitrary vertex of $T$ and $T_{c}$ be the subtree of $T$ with root $c$. We construct $A^{T}=\left[a_{r c}\right]$ by $a_{r c}=1$ if and only if there exists a vertex in $T_{c}$ with label $r$. Gusfeild (1991) showed that $A^{T}$ is a PPM. Let $r$ be a row of $A$, which is the 
label of $u$ in $T$. Similar to Lemma 2, $a_{r w}=1$ if and only if $w$ is located in a path from root to $u$. Since labels are different and there is a unique path between the root and the vertices of $T$, rows of $A$ are nonidentical.

It is obvious that $A^{T_{(A)}}=A$ and $T_{\left(A^{T}\right)}=T$. Therefore, there is a one-to-one correspondence between the set of all PPM with $k$ nonidentical rows and $n$ columns and the set of all $(k, n)$-complete tree.

\section{Extended tree}

Let $A_{k \times n}$ be an $(m, a, b)$-extendable matrix, and let the $(k, a, b)$ PPM matrix $B_{m \times n}$ be its extension and $T_{(A)}$ be its corresponding tree. Let $w$ be the repeating time function defined on the labeled vertices of $T_{(A)}$ as $w(v)=t$ if and only if $v$ is labeled by $r$ and row $r$ is repeated $t$ times in $B$. We call $\left(T_{(A)}, w\right)$ the $(m, a, b)$ extended tree of $A$ and $w(v)$ the repeating label of $v$.

Lemma 3. The MAF of column $c$ in $B$ is the sum of the repeating label of the vertices in $T_{c}$.

Proof. Let $a_{r c}=1$. Then, by Lemma 3.2, $c$ is located in a path from root to vertex $v$ with label $r$. So, $v$ is a vertex of $T_{c}$. Let $w(v)=t$. Corresponding to $t$ repeats of $r$, we have $t$ ones in column $c$. Therefore, the MAF of column $c$ is equal to the sum of repeating labels in $T_{c}$.

Example 3. For matrices in Example 2, repeating labels and MAFs are shown in Figure 3 . Let $A_{k \times n}$ be an $(m, a, b)$ extendable matrix and $\left(T_{(A)}, w\right)$ be the $(m, a, b)$-extended tree of $A$. Now, by the previous lemmas, we have the following observations.

\section{Observations:}

$O_{1}$ : Let $u$ be the ancestor of $v$ in $\left(T_{(A)}, w\right)$; then the MAF of column $u$ is greater than or equal to the MAF of column $v$.

$\mathrm{O}_{2}$ : Let $v$ be a leaf with label $i$ in $\left(T_{(A)}, w\right)$. By proof of Lemma 3.1, column $v$ in $A_{k \times n}$ has only 1 nonzero entry in row $i$. Since the MAF of column $v$ should be at least $a, w(v) \geq a$.

\section{Repeating labels}

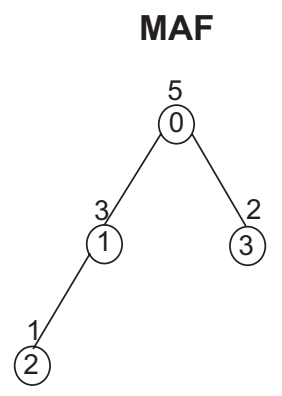

Figure 3 Repeating labels and minimum allele frequency (MAF).
$O_{3}$ : Let $T_{u_{i}}$ have $l_{i}$ leaves and $c_{i}$ labeled internal vertices. By using Lemma 4.1 and $O_{2}$, the MAF of column $u_{i}$ in $B$ is at least $a l_{i}+c_{i}$.

$O_{4}$ : Let $T_{u_{i}}$ have $l_{i}$ leaves and $c_{i}$ labeled internal vertices. Since the MAF of each column in $B$ is at most $b, O_{3}$ implies that $l_{i} \leq b / a$.

$O_{5}$ : Let $x_{1}, x_{2}, \ldots, x_{d}$ be the children of root $r$ of $\left(T_{(A)}, w\right)$. Then, using $O_{1}$ for each labeled vertex $x_{i}$, we have $w\left(x_{i}\right) \leq b$ if and only if $w\left(x_{i}\right) \leq b$ for every labeled vertex of $\left(T_{(A)}, w\right)$.

In the following theorem, we show that we can always assume that the desired matrix has at least one all-zero row. By the MAF of vertex $v$ in $T_{(A)}$, we mean the MAF of column $v$ in $A^{T}$.

Theorem 1. There is an $(m, a, b)$-extendable matrix $A_{k \times n}$ if and only if there is an $(m, a, b)$-extendable matrix $A_{k \times n}^{\prime}$ that has a zero row.

Proof. It is obvious that root $r$ of $T_{(A)}$ is labeled when $A$ has a zero row.

Let $A$ has no zero rows and $r$ is not labeled. We consider two cases:

1. There exists an internal node $u$, which is labeled by $p$. In this case, we consider the labeled tree $T^{\prime}$ by removing label $p$ of $u$ and giving $p$ to $r$.

2. Let only the leaves of $T_{(A)}$ be labeled. Consider vertex $x$ such that degree $x$ in $T_{x}$ is at least 2 , and in $T_{x}$, every vertex of $T_{x}-x$ has at most 1 child (as $k \geq 2$ and there is no labeled internal node and $T_{(A)}$ has at least two leaves, there exists such $x$ ). Let $u$ and $v$ be two leaves of $T_{x}$ and $z$ be the ancestor of $v$ and the child of $x$. (If $v$ is a child of $x$ let $z=v$.) Since $u$ is a leaf of $T_{(A)}$, it has a label such as $p$. By removing edge $x z$ from $T_{(A)}$, labeling $p$ from $u$, adding edge $u z$, and giving $p$ to $r$, we obtain a new labeled tree $T^{\prime}$ (Figure 4). In both cases, we define repeating time function $w^{\prime}$ on the labeled vertices of $T^{\prime}$ by

$$
w^{\prime}(v)=\left\{\begin{array}{cc}
w(v) & v \neq u \\
0 & v=u \\
w(u) & v=r
\end{array}\right.
$$
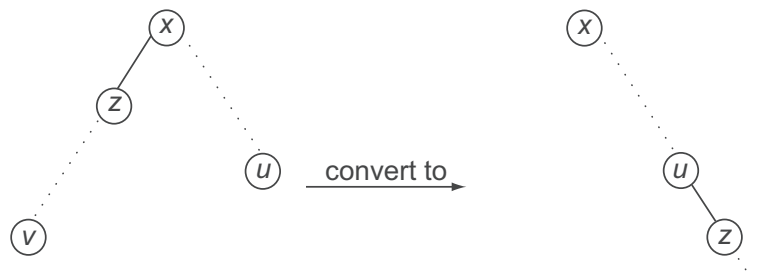

Figure $4 \mathrm{New}$ labeled tree $T^{\prime}$ obtained from $T$. 
It is obvious that $T^{\prime}$ is a $(k, n)$-complete tree, which has a zero row. Now, by using $O_{l}, \ldots, O_{5}$ and Lemma 3 , we conclude that $A^{T \prime}$ is $(m, a, b)$-extendable.

\section{Necessary conditions}

In this section, we describe some necessary conditions for the existence of an $(m, a, b)$-tree. By applying Theorem 1, we find the conditions necessary for the existence of an $(m, a, b)$-tree in which the root has been labeled. First, we introduce some necessary conditions, and then in the next section, we will show that these conditions are also sufficient.

Theorem 2. Assume that $T$ is a $(k, n)$-complete tree and $(T, w)$ is an $(m, a, b)$-tree in which the root has been labeled. Let $r$ be the root of $T, \operatorname{deg}_{T}(r)=d$, and $T$ has $l$ leaves. Then

1. $l \leq k-1$.

2. $\frac{k+(a-1) l-1}{b} \leq d \leq l$.

3. $l(\mathrm{a}-1)+k m \leq$.

Proof.

1. Since $r$ and the leaves of $T$ are labeled with nonidentical rows, 1 holds.

2. It is obvious that $d \leq l$. By $O_{2}$ for each leaf $v, w(v) \geq$ $a$. Suppose $x_{1}, x_{2}, \ldots, x_{d}$ are the children of $r$, and $T_{x_{i}}$ contains $l_{i}$ leaves and $c_{i}$ internal labeled vertices. Then by $O_{3}$, we have

$$
l_{i} a+c_{i} \leq b, \quad 1 \leq i \leq d .
$$

Therefore

$$
\begin{aligned}
& \sum_{i=1}^{d}\left(a l_{i}+c_{i}\right) \# b d \\
& a l+k-l-1 \leq b d
\end{aligned}
$$

So,

$$
\frac{k+(a-1) l-1}{b} \# d
$$

3. By $O_{2}$ for each leaf $v$, we have $w(v) \geq a$, and for each labeled vertex $u$, we have $w(u) \geq 1$.

Then, the number of rows in a $(k, a, b)$-PPM matrix $B_{m \times n}$, which is an extension of extended $A_{T}$, is at least

$$
a l+(k-l) .
$$

We categorize the children of $r, x_{1}, x_{2}, \ldots, x_{d}$, into 3 groups:

- $A_{1}=\left\{\xi_{l} \mid \chi_{t}=0 \alpha v \delta \lambda_{l}=1\right\}$

- $A_{2}=\left\{\xi_{l} \mid \chi_{t}=0 \alpha v \delta \lambda_{t} \neq 1\right\}$

- $A_{3}=\left\{\xi_{1} \mid \chi_{t} \neq 0\right\}$

Let $\alpha=\left|A_{1}\right|$ and $\beta=\left|A_{2}\right|$.
Theorem 3. Let $(T, w)$ be the same as in Theorem 2 and $B_{m \times n}$ is its corresponding $(k, a, b)$-PPM. Then

1. $l_{i} \leq b / a$

2. $d+l-n \leq a$

3. Let $a / b$ ( $a$ be a divisor of $b$ ) and $a \neq b$; then the number of $x_{i}$ in which $T_{x_{i}}$ has $b / a$ leaves is less than or equal to $n-k+1$.

Proof.

1. It results from the observation $\mathrm{O}_{4}$.

2. Let $n_{i}$ be the number of vertices of $T_{x_{i}}$. Then, obviously

$$
n_{i} \$ \begin{cases}c_{i}+l_{i} & c_{i} \geq 1 \\ l_{i}+1 & c_{i}=0 \text { and } l_{i} \neq 1 \\ 1 & c_{i}=0 \text { and } l_{i} \neq 1\end{cases}
$$

So, we have

$$
\begin{aligned}
n & =\sum_{i=1}^{d} n_{i} \\
& =\sum_{c_{i} \geq 1} n_{i}+\sum_{c_{i}=0, l_{i} \neq 1} n_{i}+\sum_{c_{i}=0, l_{i}=1} n_{i} \\
& \geq \sum_{c_{i} \geq 1}\left(c_{i}+l_{i}\right)+\sum_{c_{i}=0, l_{i} \neq 1}\left(l_{i}+1\right)+\sum_{c_{i}=0, l_{i}=1} 1 \\
& =\sum c_{i}+\sum l_{i}+\beta
\end{aligned}
$$

Now, we find the upper and lower bounds.

- Upper bound

$$
\begin{aligned}
& n \geq \sum c_{i}+\sum l_{i}+\beta \\
& \quad=k-l-1+l+\beta \\
& \Rightarrow \beta \leq n-k+12
\end{aligned}
$$

\section{- Lower bound}

We have $\left|A_{1} \cup A_{2}\right|=d-a$. Since the number of internal vertices of $T$ which have labels is $k-l-1$,

$$
\begin{gathered}
\beta \geq(d-\alpha)-(k-1-1) \\
=d-\alpha+l-k+1
\end{gathered}
$$

Now, we have

$$
\begin{gathered}
d-\alpha-k+l-1 \leq n-k+1 \\
d+l-n \leq \alpha
\end{gathered}
$$

and part 2 is thus proved.

3. Suppose $a / b$ and $a \neq b$. Let $S=\left\{x_{i} \mid l_{i}=b / a\right\}$. By observation $O_{3}$, for each $x_{i} \in S$, we have $c_{i}=0$. So, $S \subset A_{2}$, and by inequality 1 , we have $|S| \leq n-k+1$.

\section{Sufficient conditions}

In the previous section, we obtained some necessary conditions for the existence of an $(m, a, b)$-extendable tree whose root has been labeled. In this section, we show that these 


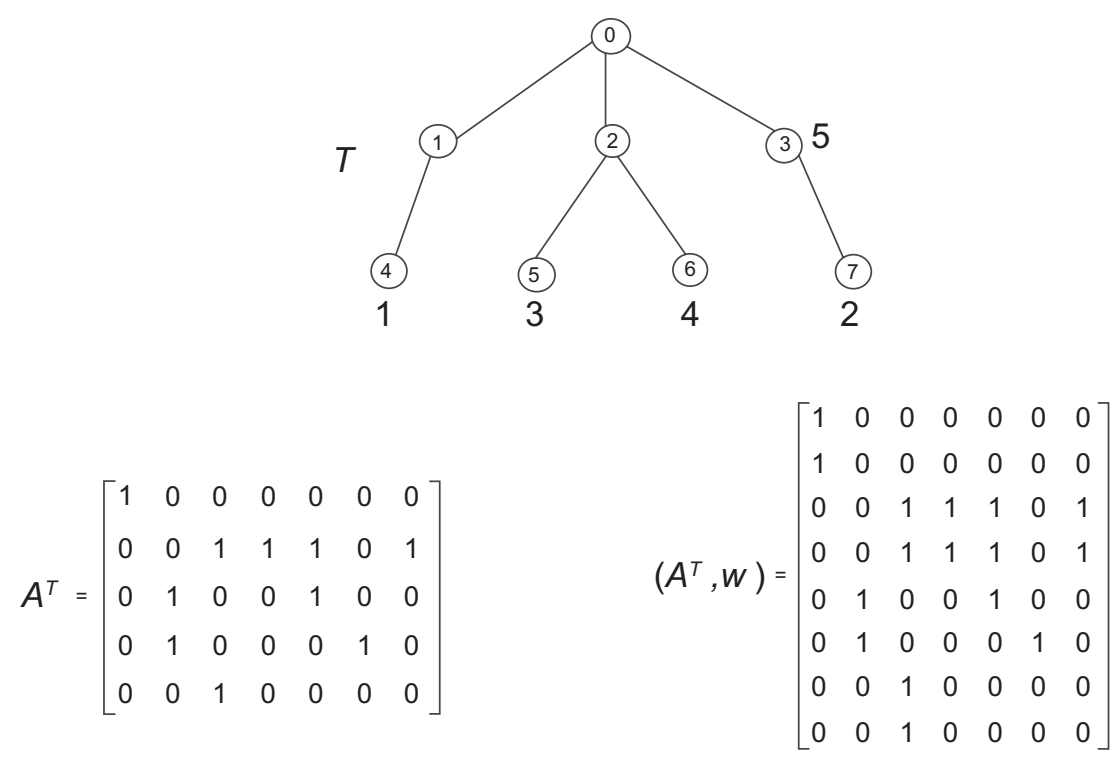

Figure 5 In this figure, we have $w(1)=w(2)=w(5)=2$ and $w(3)=w(4)=1$.

conditions are sufficient too. For this purpose, let $l_{1}, l_{1}, \ldots$, $l_{d}$ satisfy the conditions of Theorems 2 and 3 . Then, we introduce an algorithm for constructing the rooted $(m, a, b)$ extendable tree $T$ with root $r ; x_{1}, \ldots, x_{d}$ are the children of $r$ and $T_{x_{i}}$ has $l_{i}$ leaves. First, we determine the number of labeled internal nodes in each $T_{x_{i}}$.

\section{Determination of $\boldsymbol{c}_{\boldsymbol{i}}$}

We categorize the children of $r$ into three groups:

1. $G_{1}$ : Children with $l_{i}=1$. By Theorem 3, part 2 , we have $\left|G_{1}\right| \geq d+l-n$.

2. $G_{2}$ : Children with $l_{i}=b / a$ and $l_{i} \neq 1$.

3. $G_{3}$ : Children with $l_{i}=b / a$ for $b \neq a$. By Theorem 3, part 3, we have $\left|G_{3}\right| \geq n-k+1$.

According to $G_{1}, G_{2}$, and $G_{3}$, we determine $c_{i}$ s as follows:

1. For each $x_{i} \in G_{2}$ and $d+l-n$ elements of $G_{1}$, we set $c_{i}=0$.

2. Let $\left|G_{2}\right| \leq k-l+1$. Then, for each $x_{i} \in G_{2}$, we assign $c_{i}=1$. Now, we distribute the value $k-l+1-\left|G_{2}\right|$ among the members of $G_{2}$ and those of $G_{1}$ for which $c_{i} \neq 0$ or $c_{i}$ is not determined in step 1. Now, suppose $\left|G_{2}\right| \geq k-l+1$. Let $F$ be a subset of $G_{2}$ of size $k-l+1$. For each $x_{i} \in F$, set $c_{i}=1$. For all the remaining vertices such as $x_{j}$, which is not considered in the above steps, we assign $c_{j}=0$. By this procedure, part 2 of the Theorem 3, $\left(\left|A_{1}\right| \geq d+l-n\right)$ holds.

\section{Algorithm for determination of $c_{i}$}

1. Categorize $d$ children of the root
2. for $i=1$ to $d$

3. if $l_{i}=1$ then put this child in $G_{1}$

4. if $l_{i} \neq b / a$ and $l_{i} \neq 1$ then put this child in $G_{2}$

5. if $l_{i}=b / a$ and $b \neq \mathrm{a}$ then put this child in $G_{3}$

6. determine $c_{i}$ (related to each $x_{i}$ ) according to $G$

7. if $x_{i} \in G_{3}$ or $d+l-n \in G_{1}$

8. $c_{i}=0$

9. else if $\left|G_{2}\right| \leq k-l+1$

10. if $x_{i} \in G_{2}$ then

11. $c_{i}=1$

12. Distribute $k-l+1-\left|G_{2}\right|$ among members of $G_{2}$ and those of $G_{1}$ for which $c_{i} \neq 0$

13. else if $\left|G_{2}\right|>k-l+1$

14. $F \leftarrow$ (subset of $G_{2}$ of size $\left.k-l+1\right)$

15. if $x_{i} \in F$ then $c_{i}=1$

16. else $\mathrm{c}_{1}=0$.

\section{Determination of $\boldsymbol{n}_{\boldsymbol{i}}$}

- We first initialize $n_{i}$ for each $x_{i}$ as follows:

$$
n_{i}= \begin{cases}c_{i}+l_{i} & c_{i} \geq 1 \\ l_{i}+1 & c_{i}=0 \text { and } l_{i} \neq 1 \\ 1 & c_{i}=0 \text { and } l_{i}=1\end{cases}
$$

- We distribute $n-\sum n_{i}$ between all $T_{x_{i}}$ at random.

To show that these steps are possible, it is enough to show that $\sum n_{i} \leq n$. By the proof of part 2 of Theorem 3, it is enough to show that $\beta \leq n-k+1$. The number of vertices for which $l_{i} \neq 1$ and $c_{i}=0, \beta$, is as follows: 
1. If $\left|G_{2}\right|<k-1+1$, then $\beta=\left|G_{3}\right|$.

2. If $\left|G_{2}\right| \geq k-l+1$, then $\beta=\left|G_{3}\right|+\left|G_{2}\right|-k+l-1$.

Since $\left|G_{3}\right|+\left|G_{2}\right|=d-\left|G_{1}\right|$ and $\left|G_{1}\right| \geq d+l-n$, $\left|G_{3}\right|+\left|G_{2}\right| \leq n-l$, in both cases, $\beta \leq n-k+1$.

Algorithm for the determination of $\boldsymbol{n}_{\boldsymbol{i}}$

1. Initialize $n_{i}$ for each $x_{i}$

2. if $c_{i} \geq 1$

3. then $n_{i}=c_{i}+l_{i}$

4. else if $c_{i}=0$ and $l_{i} \neq 1$

5. then $n_{i}=l_{i}+1$

6. else if $c_{i}=0$ and $l_{i}=1$

7. then $n_{i}=1$

8. Distribute $n-\sum n_{i}$ between trees related to children of the $\operatorname{root}\left(T_{x_{i}}\right)$

\section{Constructing $(m, a, b)$-extendable tree}

In this subsection, we will construct a rooted tree $T$ with root $r . x_{1}, \ldots, x_{d}$ are its children, and $T_{x_{i}}$ has $n_{i}$ vertices, $l_{i}$ leaves, and $c_{i}$ labeled internal vertices for which $c_{i} \mathrm{~s}$ and $n_{i} \mathrm{~s}$ are determined as described earlier. The following algorithm constructs $T_{x_{i}}, 1 \leq i \leq d$.

Algorithm for the construction of $T_{i}\left(n_{i}, l_{i}, c_{i}\right)$

1. Let $L S$ be the set of leaf vertices

2. Let $I S$ be the set of internal vertices

3. $I S \leftarrow x_{i}$

4. $L S \leftarrow \phi$

5. for $j \leftarrow 2$ to $n_{i}$

6. do if sizeof $(L S)=l_{i}$

7. then $P S=L S$

8. else if $l_{i}-$ sizeof $(L S)=n_{i}-j+1$

9. then $P S=I S$

10. else $P S=L S \cup I S$

11. select vertex $v$ from $P S$ randomly and put the new vertex, $w$, as $v^{\prime}$ s child

12. $L S=L S \cup w$

13. if $v \in L S$

14. then $L S \leftarrow L S-v$

15. $I S \leftarrow I S \cup v$

16. Mark $c_{i}$ vertices from $I S$.

Now, we add the root $r$ and edges $r x_{i}, 1 \leq i \leq d$. The desired tree $T$ is constructed. To label the vertices of $T_{x_{i}}$, $1 \leq i \leq d$ and root $r$ and the leaves of $T$, we assign $\{1,2, \ldots, k\}$ to the labeled vertices of $\bigcup_{i} T_{x_{i}}$ randomly.

In this algorithm, we first construct all ordered pairs $(l, d)$ that satisfy the conditions of Theorems 2 and 3. Then, we choose some of these pairs randomly and construct all $d$-tuples $\left(l_{1}, \ldots, l_{d}\right)$, satisfying the conditions of
Theorems 2 and 3 and $l=l_{1}+l_{2}+\ldots+l_{\mathrm{d}}$. Now, one of the $d$-tuples is chosen randomly. Then, we classify $x_{i}$ according to $l_{i}$. Using this classification, we consider a primary class for $c_{i}$. Now, for the remaining vertices for which we have not assigned any $c_{i}$, we choose a $c_{i}$ randomly. For calculating $n_{i}$, we first assign an initial value for each vertex $x_{i}$ and then distribute $n-\sum n_{i}$ randomly. It should be noted again that by randomness, we mean distribution according to a uniform random variable.

We also define a function $w$ on the labeled vertices of the $(k, n)$-complete tree, $T$, such that $(T, w)$ becomes an ( $m, a, b)$-tree (Figure 5). We obtain $w$ from the following recursive algorithm:

$$
w_{o}(u)= \begin{cases}a & \text { if } u \text { is a leaf of } T \\ 1 & \text { if } u \text { is a labled vertex }\end{cases}
$$

We define $w_{i+1}$ recursively from $w_{i}$ as follows: If there exists $x_{j}$ such that the MAF of $x_{j}$ in $\left(T, w_{i}\right)$ is less than $b$, we choose an arbitrarily labeled vertex from $T x_{\mathrm{i}}$, such as $u$, and define $w_{i+1}(u)=w_{\mathrm{i}}(u)+1$. We continue this procedure until we obtain the function $w_{j}$ such that $\sum_{u \in T} w_{j}(u)=m$ or the MAF of $x_{i}=b$ for all $1 \leq i \leq d$. Now, $w$ is defined by $w=w_{j}$ if $\sum_{u \in T} w_{j}(u)=m$. For the case that $\sum_{u \in T} w_{j}(u)<m$, we consider $w$ by

$$
w(u)= \begin{cases}m-\sum_{u \in T} w(u) & \text { if } u=r \\ w(u) & \text { if } u \neq r\end{cases}
$$

Let the vertex $u$ of $A_{T}$ have labels $r_{0}$ and $w(u)=0$. We repeat row $r_{0}$ of $A_{T}, t$ times. Let $B$ be the matrix obtained from $A_{T}$ by repeating the procedure. It is obvious that $B$ has $m$ rows, $n$ columns, and $k$ nonidentical rows. Let $u$ be a column of $B$ and a vertex of $(T, w)$. Consider $T_{u}$ and one of its leaf, such as $u_{0}$, with label $r_{0}$. We know that $w\left(u_{0}\right) \geq a$. The entry of $A_{T}$ in column $u_{0}$ and row $r_{0}$ is 1 . Therefore, by Lemma 2, the entry of $A_{T}$ in column $u$ and row $r_{0}$ is also 1 . Since we repeat row $r_{0}$ in $B$ at least $a$ times, the MAF of column $u$ in $B$ is at least $a$. On the other hand, let $x_{i}$ be the ancestor of $u$. Since $w(u) \leq w\left(x_{i}\right)$ and $\operatorname{MAF}\left(x_{\mathrm{i}}\right) \leq b$ by observation $O_{l}$, $M A F(u) \leq b$. Therefore, $B_{m \times n}$ is a $(k, a, b)$-PPM.

\section{Running time of the algorithm}

This algorithm has five steps. In the first step, the algorithm finds $d, l$, and $l_{l}, \ldots, l_{d^{\prime}}$ which satisfy the necessary conditions in $O\left(n^{2}\right)$. In the second step, the algorithm finds the $n_{i}$ and $c_{i}$ for $1 \leq i \leq d$. In the third step, the algorithm constructs $T x_{i}, 1 \leq i \leq d$, with $n_{i}$ internal vertices, $c_{i}$ labeled internal 
vertices, and $l_{i}$ in $O\left(n_{i}^{2}\right)$. As $\sum_{i=1}^{d} n_{i} \leq n$ and $\sum_{i=1}^{d} n_{i}^{2} \leq n^{2}$, the running time of the algorithm in this part is $O\left(n^{2}\right)$. In the next step, the algorithm defines a function on labeled vertices and finds its value recursively. As this function is called at most $m$ times and in each call updating MAF of $x_{i}^{\prime}$ s takes $O(n)$, the running time of the algorithm in this part is $O(m n)$. In the last step, the algorithm constructs the desired matrix from the tree in $O(m n)$. Thus, the total running time of the algorithm is $O\left(\max \left(n^{2}, n m\right)\right)$.

\section{Discussion}

In this article, we have presented a new model for perfect phylogeny matrices. Our goal was to construct a random perfect phylogeny matrix with $k$ different haplotypes, $n$ SNPs, and a population size of $m$ for which the MAF of each SNP is between two specific numbers $a$ and $b$. Our new approach allows us to find the necessary and sufficient conditions for the existence of such a matrix. As the solution matrix is a binary matrix with $m$ rows and $n$ columns, any algorithm for this problem is $\Omega(\mathrm{nm})$. We developed an $O\left(\max \left(n^{2}, n m\right)\right)$ time algorithm based on this model to solve this problem.

We used the available methods to construct the perfect phylogeny matrix without taking MAF into account, and we then eliminated those columns that do not satisfy the MAF condition. It should be noted that there are two problems concerning this approach. First, we need to use an algorithm that is able to calculate the MAF of each column automatically and eliminate it if the conditions are not satisfied. Second, it is very probable that the number of columns that should be removed is very high. So, we will obtain matrices with few columns, and we have to run the algorithm several times to obtain a matrix with $n$ columns and the required MAF. Therefore, an algorithm that could construct such matrices is of much interest. We have developed software, RAPPER, for implementing this algorithm, which is available at http://bioinf.cs.ipm. ir/softwares/RAPPER.

\section{Acknowledgment}

Changiz Eslahchi thanks Shahid Beheshtshi University for their support. This research is in part supported by a grant from IPM.

\section{Disclosure}

The authors report no conflicts of interest in this work.

\section{References}

1. Gusfield D. Haplotyping as perfect phylogeny: conceptual framework and efficient solutions. In: Myers G, Hannenhalli S, San-koff D, et al. editors. Proceedings of RECOMB 2002. New York, NY: ACM Press; 2002:165-175.

2. Bafna V, Gusfield D, Lancia G, Yooseph S. Haplotyping as perfect phylogeny: a direct approach. J Comput Biol. 2003;10:323-340.

3. Eskin E, Halperin E, Karp R. Efficient reconstruction of haplotype structure via perfect phylogeny. J Bioinform Comput Biol. 2003;1:1-20.

4. Excoffier L, Novembre J, Schneider S. SIMCOAL: a general coalescent program for the simulation of molecular data in interconnected populations with arbitrary demography. J Heredity. 2000;91:506-509.

5. Spencer CC, Coop G. SelSim: a program to simulate population genetic data with natural selection and recombination. Bioinformatics. 2004;20:3673-3675.

6. Mailund T, Schierup MH, Pedersen CN, Mechlenborg PJ, Madsen JN, Schauser L. CoaSim: a flexible environment for simulating genetic data under coalescent models. BMC Bioinformatics. 2005;6:252.

7. Marjoram P, Wall JD. Fast "coalescent" simulation. BMC Genet. 2006;7:16.

8. Hudson RR. Generating samples under a Wright-Fisher neutral model of genetic variation. Bioinformatics. 2002;18:337-338.

9. Hellenthal G, Stephens M. msHOT: modifying Hudson's ms simulator to incorporate crossover and gene conversion hotspots. Bioinformatics. 2007;23:520-521.

10. Posada D, Wiuf C. Simulating haplotype blocks in the human genome. Bioinformatics. 2003;19:289-290.

11. Montanna G. HapSim: a simulation tool for generating haplotype data with pre specified allele frequencies and LD coefficients. Bioinformatics. 2003;21:4309-4311.

12. Gusfield D. Efficient algorithms for inferring evolutionary trees. Networks. 1991;21:19-28.

13. Pe'er I, Pupko T, Shamir R, Sharan R. Incomplete directed perfect phylogeny. SIAM J Comput. 2004;33:597-607.

14. Estabrook GF, Johnson CS Jr, McMorris FR. An idealized concept of the true cladistic character. Math Biosci. 1975;23:263-272.

15. Meacham C. Theoretical and computational considerations of the compatibility of qualitative taxonomic characters. Numerical Taxonomy. Nato ASI Series. 1983;G1:304-314.

\section{Publish your work in this journal}

Advances and Applications in Bioinformatics and Chemistry is an international, peer-reviewed open-access journal that publishes articles in the following fields: Computational biomodelling; Bioinformatics; Computational genomics; Molecular modelling; Protein structure modelling and structural genomics; Systems Biology; Computational

\section{Dovepress}

Biochemistry; Computational Biophysics; Chemoinformatics and Drug Design; In silico ADME/Tox prediction. The manuscript management system is completely online and includes a very quick and fair peerreview system, which is all easy to use. Visit http://www.dovepress.com/ testimonials.php to read real quotes from published authors. 